\title{
Investigating Alopecia Areata and its Connection to The High-Mobility Group Box 1: Review Article
}

\author{
Asmaa Abd El-Hameed El-Sayed*, Afaf Helmy Ghanem, Mohamed Ibrahim El Ghareeb \\ Dermatology, Venereology and Andrology Department, Faculty of Medicine, Zagazig University, Egypt \\ *Corresponding Author: Asmaa Abd El-Hameed El-Sayed, E-Mail: abdelhamied74@gmail.com
}

\begin{abstract}
Background: It is a common and reversible hair loss illness known as alopecia areata (AA). Hair loss on the scalp and other regions of the body can begin as a patchy area of full hair loss which may develop to the entire loss of all hair on the body. It is uncertain what causes AA but it is characterized by hair cycle disruption and the presence of mononuclear cell infiltrates in the perifollicular, as well as peribulbar areas. There have been numerous studies that have shown that AA has been linked to various autoimmune illnesses, including vitiligo. Autoimmune disease is becoming more prevalent, and researchers have found a link between chronic inflammation and HMGB1 (high-mobility group box 1). Objective: To evaluate the role of (HMGB1) protein in pathogenesis of AA. Conclusion: HMGB1 is a promising predictor of prognosis and treatment responsiveness in the pathophysiology of alopecia areata.
\end{abstract}

Keywords: Alopecia Areata, High-Mobility Group Box 1.

\section{INTRODUCTION}

Autoimmune, and inflammatory disease alopecia areata is characterized by non-scarring hair loss. There are numerous clinical symptoms of alopecia areata. If you have hair, your scalp is the most likely area to be affected. Alopecia areata is a condition that results in the whole or partial loss of hair on a specific area of the body or on the entire body. Most hair loss is circumscribed patches, but it can also progress to include the entire scalp (alopecia totalis) or involve the entire body (alopecia universalis). Hair loss can manifest in any of these ways ${ }^{(\mathbf{1})}$.

Most patients with alopecia areata are asymptomatic; however, it can cause significant psychosocial stress in those with severe or chronic forms of the illness. Localized, well-defined patches of hair loss are the most common symptoms. The condition can manifest in various ways: as a single patch of hair loss or as multiple patches of hair loss, including alopecia totalis, alopecia reticularis, alopecia sisaipho (inverse ophiasis), as well as alopecia universalis, all of which cause complete hair loss on the scalp or even the entire body, as well as ophiasis, which affects only the sides ${ }^{(2)}$.

The protein HMGB1 is highly conserved in the nucleus. Cell nuclei use it to control recombination, transcription, repair, and replication of DNA. It is also possible for cells to secrete HMGB1 into the extracellular matrix to serve as a signaling molecule in response to stimuli. Cells that have died, are dying, or have been wounded emit HMGB1 passively into the environment. Extracellular HMGB1 is therefore seen as being an ideal "necrosis marker" released by the immune system to detect tissue destruction ${ }^{(3)}$.
The aim of the present review was to detect the role of (HMGB1) protein level in pathogenesis of AA.

\section{Epidemiology:}

About 0.1-0.2 percent of the population suffers from alopecia areata. Children, men, and women of all hair colours are affected by the disease. Although the disease is rare in children before the age of three, most patients are between the ages of two and six. Between the ages of 30 and 40, the greatest number of people suffer from the disease. Only $20 \%$ of patients are over the age of 40, while $66 \%$ are under the age of $30^{(4)}$. AA appears to impact both sexes equally. According to certain surveys, the adult age group is dominated by men, while others report different findings ${ }^{(5)}$.

\section{Etiopathogenesis:}

Alopecia's etiopathogenesis is uncertain. People with a history of alopecia areata are suffering from an illness that is both a symptom and a sickness in and of itself, caused by a variety of complex etiological reasons (6). People who are genetically susceptible to AA are thought to suffer from T-cell-mediated autoimmunity. Predisposition genetic and environmental variables, in addition to immunological dysfunction, have a significant role in the progression of the disease. Trace element changes, viruses, thyroid dysfunction, as well as endocrine abnormalities, have also been postulated to play a role in perifollicular nerves and vasculature. A lack of trace elements may lead to the start of AA, according to some theories ${ }^{(7)}$.

\section{Classification of alopecia areata (Figure 1):}


The most frequent form of AA is patchy, which affects up to $75 \%$ of individuals. There are a number of active, persistent, and/or regrowing patches of alopecia reticularis, which may blend into a mosaic pattern. Alopecia totalis "AT" affects $10 \%$ to $20 \%$ of individuals, while alopecia universalis (AU) affects the rest of the body and affects the scalp. Phenomenal band-like pattern that runs from the hairline to the temples has been named "ophiasis type" by dermatologists. In most cases, it has a poor prognosis and is difficult to cure. Sisaipho is the opposite of ophiasis type, when hairs are shed in the center of the scalp and spared in the perimeter. Although widespread AA affects all hair follicles on the scalp, it usually does not impact every single one of them ${ }^{(2)}$.

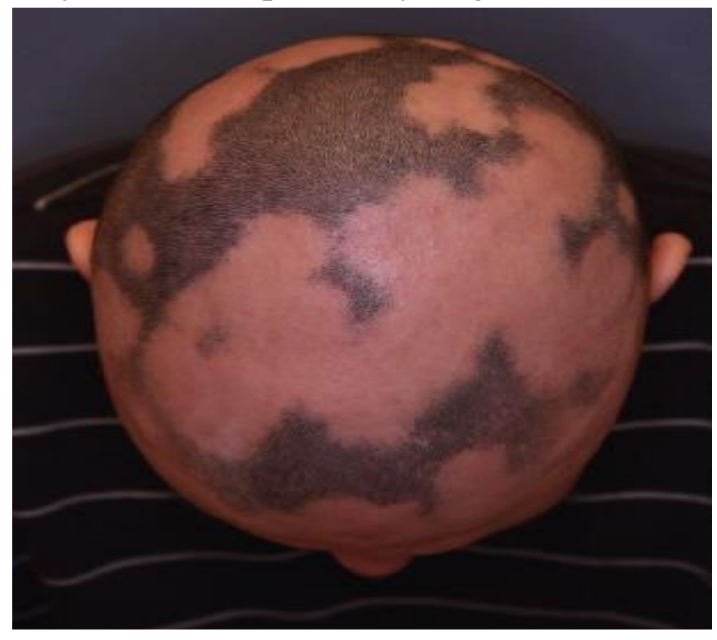

Alopecia areata reticularis.

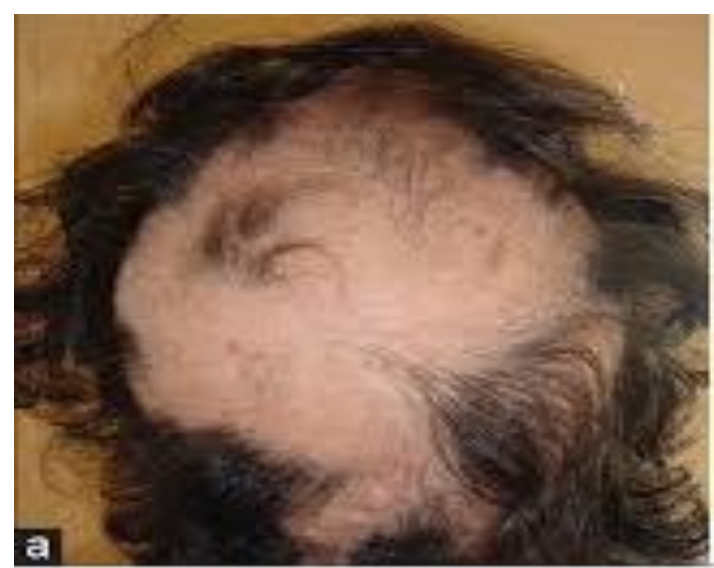

Alopecia areata sisaipho type.

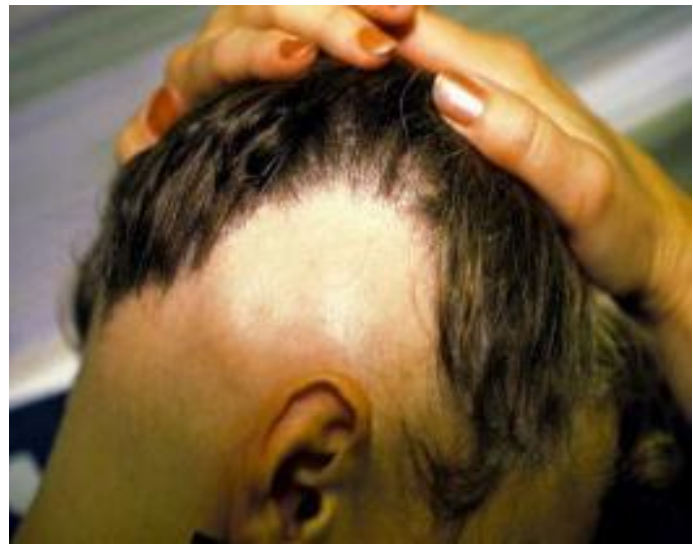

Alopecia areata ophiasis type.

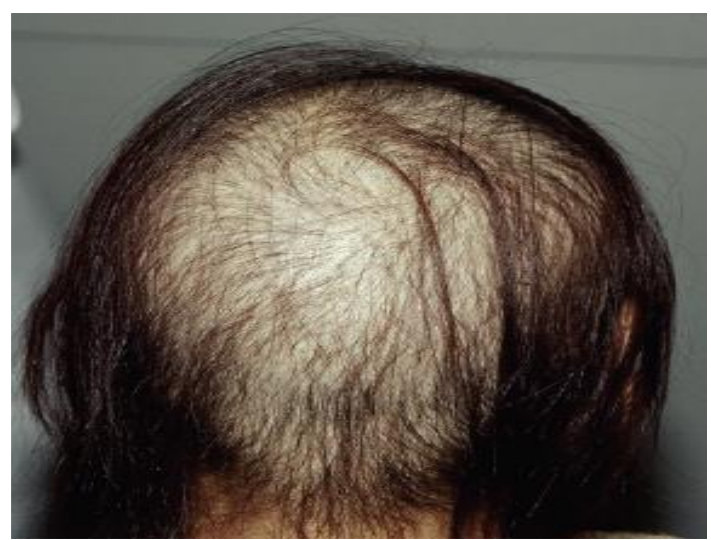

Alopecia areata, diffuse type.

Figure (1): Clinical types of $\mathrm{AA}^{(8)}$.

\section{Clinical picture:}

Round or oval patches of non-scarring hair loss arise suddenly, with remissions and exacerbations occurring on their own. With a gentle peachy hue and exclamation point hairs around the edge, the patches are well-defined. The distal segment of exclamation point hairs is wider than the proximal end of short broken hairs. It is common for the affected skin to be silky smooth and nearly always hair free ${ }^{(\mathbf{8})}$. Scalp hair, as well as body including eyelashes, beard, eyebrows, pubic and axillary hair, might be impacted, but the scalp is the most prevalent region (90 percent) ${ }^{(9)}$. Patients rarely experience any symptoms prior to hair loss; however, some do experience itching, burning, or pain. There may be a positive hair pull test in the periphery of alopecia lesions in active disease. In addition to hair loss, another symptom of AA is nail irregularities, which can occur before or after the disease begins, and they normally go away on their own, but they can continue for a long time after hair recovery has been complete ${ }^{(\mathbf{1 0})}$.

\section{Role of HMGB1 in alopecia areata:}

The most common cause of inflammation-induced hair loss is alopecia areata. Despite the fact that AA's etiology remains a mystery ${ }^{(11)}$, the involvement of $\mathrm{T}$ cells and cytokines is becoming increasingly clear from the 
growing research evidence ${ }^{(\mathbf{1 2})}$. Hair follicle autoantigens are thought to be attacked by T-cells when constitutive immune privilege is lost, leading to the development of AA in previously healthy hair follicles ${ }^{(8)}$.

Alopecia areata has been linked to various autoimmune diseases, such as autoimmune vitiligo and thyroiditis, although there is increasing evidence that AA is a tissue-specific autoimmune disorder ${ }^{(13)}$. Lamore $\boldsymbol{e t}$ al. ${ }^{(14)}$ found a link between chronic inflammation and autoimmunity and high-mobility group box 1 (HMGB1).

There are large amounts of HMGB 1 in all mammalian nuclei. The protein HMGB1 is found in all living organisms. Acute lung injury, sepsis, and rheumatoid arthritis are all examples of conditions in which it is a proinflammatory cytokine. Lipopolysaccharide, and interleukin (IL)-1, tumor necrosis factor-alfa activating monocytes and released by macrophages, as well as other cell types that are activated by these factors. Passive release of HMGB1 from necrotic or late-phase apoptotic cells is another possibility ${ }^{\mathbf{( 1 5})}$.

For example, Toll-like receptors 2, 4, 9; all interact with extracellular HMGB1 to carry out its biological functions. An increasing number of researchers are looking into the connection between autoimmune illnesses and HMGB1. Systemic lupus erythematosus (SLE), Sjogren syndrome, and rheumatoid arthritis are three conditions in which elevated HMGB1 expression has been found ${ }^{(16)}$.

Studies have shown that HMGB1 expression is enhanced in cutaneous lupus erythematosus, as well as systemic sclerosis, two autoimmune diseases with cutaneous symptoms. Because AA is thought to be an autoimmune disease specific to the tissues, it is plausible that HMGB1 may also play a role in the illness's development (17). When HMGB1 is activated, chemoattractive movement of cells as well as proinflammatory cytokines release such as TNF-alfa, IL$1 \mathrm{~b}$, and IL-6, are all facilitated, as are the effects on other immune cells such as macrophages, B cells, T cells, and monocytes. TNF-alpha, interferon-g, IL-2, and IL-1b, as well as ICAM-1 and MHC molecules on hair follicle keratinocytes and dermal papilla cells, are all abnormally expressed in AA lesions ${ }^{(\mathbf{1 8})}$.

As Kasumagic-Halilovic and colleagues (19) discovered that these cytokines play an important role in the pathophysiology of AA and are found to be overexpressed in those who suffer from the disease. HMGB1 may be a key role in AA's inflammatory processes, and may form a proinflammatory loop.

\section{CONCLUSION}

In the pathophysiology of alopecia areata disease, HMGB1 is a promising predictor of prognosis and therapeutic responsiveness.

\section{Financial support and sponsorship: Nil. Conflict of interest: Nil.}

\section{REFERENCES}

1. Prie B, Voiculescu V, Ionescu-Bozdog S et al. (2015): Oxidative stress and alopecia areata. J Med Life, 8:43-46.

2. Finner A (2011): Alopecia areata clinical presentation, diagnosis and unusual cases. Dermatol Ther., 24(3): 348-354.

3. Zhang Z, Wang M, Zhou $L$ (2015): Increased HMGB1 and cleaved caspase- 3 stimulate the proliferation of tumor cells and are correlated with the poor prognosis in colorectal cancer. $\mathrm{J}$ Exp Clin Cancer Res., 34(1): 51-54.

4. Abedini R., Hallaji Z., Lajevardi V et al. (2017): Quality of life in mild and severe alopecia areata patients. International Journal of Women's Dermatology, 4: 91-94.

5. Alkhalifah A, Alsantali A, Wang $\mathbf{E}$ et al. (2010): Alopecia areata update: part I. Clinical picture, histopathology, and pathogenesis. J Am Acad Dermatol., 62: 177-188.

6. Rodriguez $T$, Fernandes $K$, Dresser $K$ et al. (2010): Concordance rate of alopecia areata in identical twins supports both genetic and environmental factors. J Am Acad Dermatol., 62(3): 525-527.

7. Bhat Y, Manzoor S, Khan A et al. (2009): Trace element levels in alopecia areata. Indian J Dermatol Venereol Leprol., 75(1):29-31.

8. Gilhar A, Kalish R (2006): Alopecia areata a tissue specific autoimmune disease of the hair follicle. Autoimmun Rev., 5(1): 64-69.

9. Wasserman D, Guzman-Sanchez D, Scott $\mathrm{K}$ et al. (2007): Alopecia areata. Int J Dermatol., 46: 121- 131.

10. Kasumagić-Halilović E (2008): Prevalence of nail abnormalities in alopecia areata. Medicinski Urnal., 14 (3): 9698.

11. Gilhar A, Etzioni A, Paus R (2012): Alopecia areata. The New England Journal of Medicine, 366(16): 1515-1525.

12. Arca E, Açikgöz G, Taştan H et al. (2004): An open, randomized, comparative study of oral finasteride and 5\% topical minoxidil in male androgenetic alopecia. Dermatology (Basel, Switzerland), 209(2): 117-125.

13. McDonagh A, Tazi-Ahnini $R$ (2002). Epidemiology and genetics of alopecia areata. Clinical and Experimental Dermatology, 27(5): 405-409.

14. Lamore S, Cabello C, Wondrak G (2010): HMGB-1 directed drug discovery targeting cutaneous inflammatory dysregulation. Curr Drug Metab., 11: 250-65.

15. Dumitriu I, Baruah P, Manfredi A et al. (2006): HMGB1: guiding immunity from within. Trends Immunol., 26: 381-7.

16. Abdulahad D, Westra J, Bijzet J et al. (2011): High mobility group box 1 (HMGB1) and anti-HMGB1 antibodies and their relation to disease characteristics in systemic lupus erythematosus. Arthritis Res Ther., 13: 71-75.

17. Yoshizaki A, Komura K, Iwata $Y$ et al. (2009): Clinical significance of serum HMGB-1 and sRAGE levels in systemic sclerosis: association with disease severity. J Clin Immunol., 29: $180-9$.

18. Yang H, Tracey K (2010): Targeting HMGB1 in inflammation. Biochim Biophys Acta., 1799: 149-56.

19. Kasumagic-Halilovic E, Prohic A, Cavaljuga S (2011): Tumor necrosis factor-alpha in patients with alopecia areata. Indian J Dermatol., 56: 494-6. 